\title{
Un método cuantitativo para medir el área de tubérculo dañado por larvas de Tecia solanivora (Lep.; Gelechiidae) a través de análisis de imágenes digitales
}

\author{
Carlos Carpio ${ }^{1 *}$, Jean-Louis Zeddam ${ }^{1,2}$, Álvaro Barragán ${ }^{1}$, Gustavo Nuñez ${ }^{3}$, \\ Marcelo Patiño ${ }^{3} \&$ Olivier Dangles ${ }^{1,2}$ \\ Escuela de Biología, Facultad de Ciencias Exactas y Naturales, Pontificia Universidad Católica \\ del Ecuador, Apartado 17-01-2184, Quito, Ecuador. \\ fccarpio@yahoo.com \\ ${ }^{2}$ IRD-LEGS and University Paris-Sud 11, Gif-sur-Yvette, Francia. \\ ${ }^{3}$ Carrera de Ingeniería en Ciencias Agropecuarias, Departamento de Ciencias de la Vida, Escuela \\ Politécnica del Ejército, Apartado 171-5-231B, Sangolquí, Ecuador.
}

Recibido: 2010-03-18, aprobado: 2010-08-30

RESUMEN.- Se desarrolló un nuevo método de evaluación del nivel de daño provocado a los tubérculos por las larvas de polillas de la papa. El método se basa en el análisis de imágenes digitales, mediante la utilización de herramientas tecnológicas como son los paquetes libres: Gimp 2.6.4 y Scion Image. Se hizo una comparación entre la metodología propuesta y la metodología convencional basada en una evaluación visual de los daños. Se encontró que el nuevo método es particularmente confiable para medir los niveles de daño intermedios que son difíciles de estimar de manera visual. Finalmente, se determinó que con el uso de la metodología propuesta se pueden obtener datos más objetivos y precisos debido a que no están sujetos a interpretaciones humanas.

PALABRAS CLAVE: Gimp 2.6.4, índice de daño, polilla de la papa, Scion Image.

ABSTRACT.- This study presents a new method to evaluate the level of tuber damage caused by potato moth larvae. This methodology was based on analyses of digital images through the use of technological tools available on the market such as the free softwares: Gimp 2.6.4 and Scion Image. A comparison was made between the proposed methodology and the conventional one based on a visual assessment of damage. It was found that the new method is particularly reliable for measuring intermediate levels of damage that are difficult to estimate visually. 

Marcelo Patiño \& Olivier Dangles

Finally, we discuss how the use of this methodology could be more objective and accurate to quantify by eliminating human interpretations.

KEY WORDS: Damage index, Gimp 2.6.4, potato moth, Scion Image.

\section{INTRODUCCIÓN}

El complejo de las polillas de la papa, Phthorimaea operculella (Zeller), Tecia solanivora (Polvony) y Symmetrischema tangolias (Gyen, 1913) (Lepidoptera: Gelechiidae), es uno de los grupos de plagas que causa mayor daño a este cultivo, tanto en campo como en almacenamiento (1). T. solanivora fue introducida a Ecuador en 1996, a través de una importación de semilla no certificada proveniente de Colombia, con destino a las zonas paperas del Carchi (2). La larva se alimenta inicialmente encima de la piel del tubérculo, para luego barrenar más profundamente y formar galerías sinuosas (3).

La estimación visual del daño causado por las plagas ha sido uno de los métodos utilizados históricamente por los investigadores y extensionistas para evaluar la eficiencia de los diferentes controles usados para el manejo de estos insectos $(4,5)$. En este tipo de evaluación se realiza una estimación visual del porcentaje de daño del tubérculo y se aplica un sistema de puntaje basado en los valores propuestos por Arias et al. (6) o Murcia y Barreto (7), con valores que van de 0 ( $0 \%$ de daño) a 4 (entre $76 \%$ a $100 \%$ de daño); el problema con este tipo de evaluación es que puede ser subjetivo y tiene limitaciones en cuanto a precisión (8).

Los avances en el procesamiento de imágenes digitales, en las metodologías usadas para los análisis y en cuanto a la tecnología de las computadoras, han exhibido un crecimiento impresionante en las pasadas décadas (9). Todo esto se constituye en tecnologías que lideran importantes áreas; por ejemplo, telecomunicaciones digitales, radiodifusión, imágenes médicas, biología, ciencias de los materiales, sistemas multimedia y sensores remotos; esto se refleja en la existencia de un gran número de publicaciones en revistas científicas internacionales, así como en libros especializados (9).

El software de dominio público SCION IMAGE es utilizado para realizar análisis de imágenes digitales y ha sido aplicado dentro de una amplia gama de novedosos métodos de investigación en agricultura aplicada, como para medir el área de las hojas, la defoliación y el impacto de insectos fitófagos sobre las hojas $(10,11)$; el porcentaje de cobertura de plagas invasivas (12); la senescencia de las hojas (13, 
14), el estado del nutriente foliar (15). Sin embargo, SCION IMAGE no había sido utilizado para estudios relacionados con tubérculos y más específicamente para análisis de daños provocados por polillas de la papa.

Los entomólogos agrícolas siempre han tenido el reto de estimar con precisión el daño causado por las plagas en los cultivos $(10,16)$. Aunque la papa (Solanum tuberosum) es uno de los principales cultivos de la región andina, sorprendentemente, no existía una metodología que permita evaluar de una manera cuantitativa y objetiva el daño provocado por las plagas en los tubérculos. Este trabajo tiene como objetivo específico el validar una metodología que se basa en el análisis de imágenes digitales para la determinación precisa del nivel de daño producido por T. solanivora,

\section{MATERIALES Y MÉTODOS}

\section{Establecimiento del ensayo}

Se utilizaron 30 cajas de cartón corrugado de $20 \mathrm{~cm}$ de ancho, $20 \mathrm{~cm}$ de largo y $25 \mathrm{~cm}$ de altura. Cada caja representó una unidad experimental donde se depositaron diez tubérculos de la variedad de papa Leona Blanca, los cuales fueron infestados con 60 huevos de $T$. solanivora. El ensayo se ubicó en una bodega del cantón Salcedo, provincia de Cotopaxi (Ecuador), a una altitud de $2.729 \mathrm{msnm}$. La temperatura media al interior de la bodega se registró usando un termómetro máximo-mínimo (Ertco, Dubuque, IA), fue de $14,5{ }^{\circ} \mathrm{C}$ $\left(\min =9,5{ }^{\circ} \mathrm{C}\right.$; máx $\left.=20^{\circ} \mathrm{C}\right)$. Después de 50 días se retiró el ensayo de la bodega y los tubérculos fueron llevados a los laboratorios de la Pontificia Universidad Católica del Ecuador en Quito para ser evaluados.

\section{Estimación visual del índice de daño provocado por T. solanivora en tubérculos de papa}

En total se evaluaron 300 tubérculos. Cada tubérculo fue cortado longitudinalmente en cuatro partes; en cada parte se estimó visualmente la intensidad del daño evaluando el área afectada por las larvas (Figura 1A). La sumatoria de daño de cada parte representa la intensidad total del daño por papa que varía entre 0 (ningún daño) y 100 (papa totalmente dañada). Luego, este porcentaje fue transformado a un índice que va de 0 a 1 (6).

\section{Adquisición de la imagen digital de los tubérculos}

Se tomaron fotografías de las cuatro partes en las que fue cortada cada una de las papas con una cámara digital Canon Power Shot S40 (Canon, Tokyo, Japón). Se usó un soporte para ubicar la cámara a una altura de 21 $\mathrm{cm}$ y un negatoscopio a manera de caja de luz para evitar las sombras. 
Como fondo, se utilizaron hojas cuadriculadas A4 $(0,5 \mathrm{~cm})$, para tener una medida conocida de referencia. Las fotos fueron exportadas a un computador. Con ayuda del programa de libre acceso GIMP 2.6.4 (Spencer Kimball, Peter Mattis and the GIMP Development Team), se estandarizó el brillo de las fotos, se definió algunos márgenes en los bordes de los tubérculos y se cambió de formato de jpg a tif para que las fotos puedan ser analizadas con el programa de libre acceso Scion Image 4.0.2. Las fotos fueron editadas para generar dos tipos diferentes de imágenes; una para calcular el área dañada de los tubérculos (Figura 1B-1) y otra en la que se invirtieron los colores para poder calcular el área total de la papa (Figura 1B-2).

\section{Procesamiento de las imágenes con Scion Image}

Para determinar el índice de daño provocado por las larvas de $T$. solanivo$r a$ se utilizó el software de dominio público Scion Image 4.0.2 para Windows (National Institute of Health, Bethesda, MD,

www.scioncorp.com/pages/scion image_windows.htm).

El programa permite analizar las imágenes en color (Figura 1B-1) o en escala de grises (Figura 1B-2). Se utilizó la versión a color para establecer la
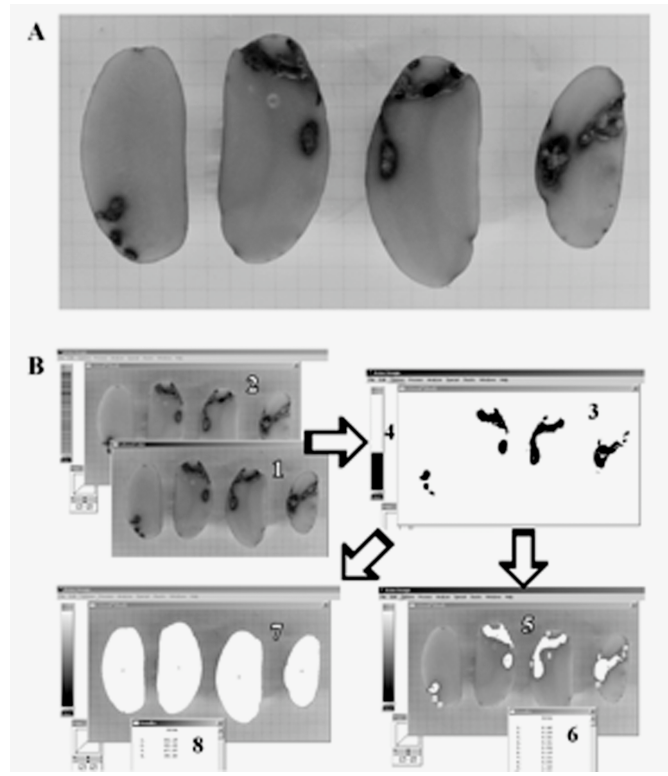

Figura 1 (A).- Foto de tubérculo cortado en cuatro partes usado para el análisis del daño provocado por larvas de T. salanivora. (B) Secuencia seguida durante el manejo del programa SCION IMAGE: (1) foto del tubérculo a color, (2) foto de la papa en escala de grises, (3) visualización del área del tubérculo dañada mediante la aplicación de la función Threshold, (4) nivel donde se ubicó la función Threshold para realizar el análisis, (50 área del tubérculo dañada, calculada (6) cuadro de los resultados correspondientes al área de daño calculada, (7) área total de papa calculada, (8) cuadro de los resultados correspondientes al área calculada.

escala real. Se utilizó la hoja cuadriculada A4 para establecer cuantos pixeles correspondían a un centímetro. Luego se escogió del menú: Analize $>$ Set scale y se ingresó la equivalencia de 1 centímetro en pixeles. Luego, se cerró la imagen a color y se continuó trabajando con la imagen en gradación de grises (Figura 1B-2). 
Posteriormente se escogió del menú: Analize $>$ Options $>$ Area $>$ OK. Luego se seleccionó del menú: Options $>$ Threshold y se comenzó a bajar el contraste hasta que el umbral llegue a 155 , una medida previamente establecida para todas las imágenes (Figura 1B$3,1 \mathrm{~B}-4)$. Con este valor se consigue el óptimo de contraste entre las zonas dañadas y las zonas sanas de la papa (en negro en la Figura 1B-3). Seguidamente, se escogió del menú: Analyze > Analyze particles y el programa procede a señalar las área que se va a calcular (Figura 1B-5). Finalmente, se escogió del menú: Analyze $>$ Show results donde apareció en un recuadro los resultados de las áreas calculadas

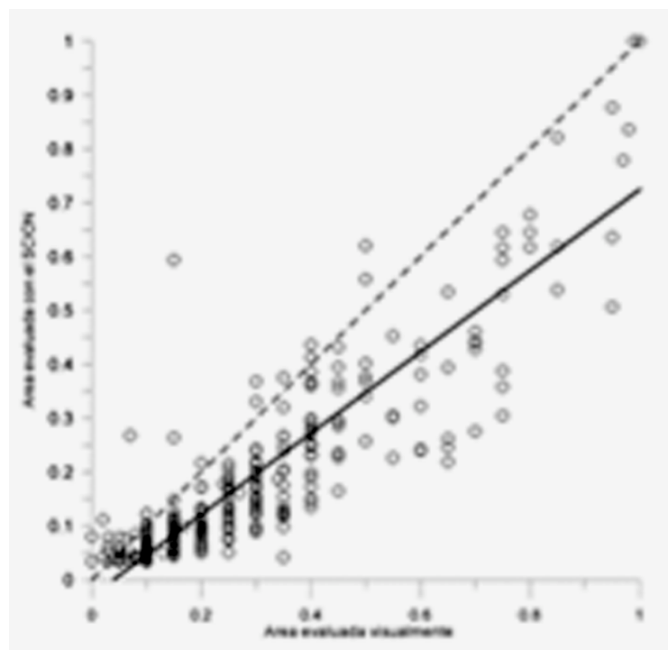

Figura 2.- Relación entre el área evaluada con el método SCION IMAGE y el área evaluada con el método visual. La línea punteada representa una línea de relación 1:1 que ayuda a visualizar mejor relación entre los dos métodos evaluados. La línea continua representa la linea de la regresión $(\mathrm{Y}=$ $0,758 * X+0.029 ; \mathrm{P}<0,001)$
(Figura 1B-6). Esta información fue copiada y exportada a una plantilla hecha con el programa Excel (Microsoft, USA). Este procedimiento se siguió para calcular el área de daño producido por las larvas (Figura 1B-5, 1B6) y un procedimiento similar se aplicó para calcular el área total de cada tubérculo (Figura 1B-7, 1B-8). Para determinar el índice de daño de cada tubérculo, se dividió el área dañada para el total fotografiado. El tiempo total para procesar cada foto fue de aproximadamente cuatro minutos.

\section{Análisis estadístico}

Para evaluar los métodos (visual vs. análisis de imágenes digitales) de estimación del daño se hizo un análisis de regresión usando Minitab (Versión 1.5). Se asumió que los datos obtenidos a través de la evaluación de las imágenes digitales corresponderían a los valores observados y los datos obtenidos a través de la evaluación visual corresponderían a los valores esperados. Siguiendo las recomendaciones de Piñeiro et al. (17), se ubicaron los valores esperados en el eje de las $\mathrm{X}$ y los valores observados en el eje de las Y. Se calculó la pendiente y la intersección.

Se calculó el coeficiente parcial de desigualdad de Theil (Usesgo, Upendiente, Uerror), el cual separa el error real de la predicción (la suma de cuadrados del error predicho), en diferentes componentes y complementa la evaluación 


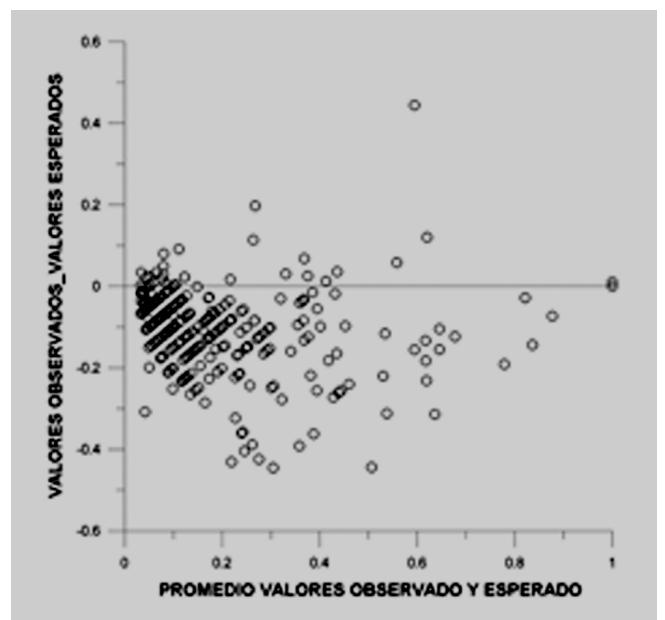

Figura 3.- Distribución del error. Los puntos representan las diferencias entre lo evaluado con el análisis visual y el análisis de imágenes digitales.

hecha con la regresión $(18,19)$. El coeficiente de Theil divide la varianza de los valores observados no explicados por los valores predichos (llamado la suma de cuadrados del error predicho), siendo Usesgo, la proporción asociada con la diferencia de las medias entre los valores observados y los valores predichos, Upendiente, la proporción asociada con la pendiente del modelo ajustado y la línea 1:1 y Uerror, es la proporción asociada con la varianza inexplicada (18).

Para observar la tendencia en la distribución del error se graficaron los valores promedio de los pares de los valores esperados y los valores observados en el eje de las abscisas y las diferencias en el eje de las ordenadas con el software Grapher (Versión 4.00). Para hacer un análisis del error se hizo una correlación de los datos obtenidos por el Scion Image y la raíz cuadrada del error medio o RMSE (la diferencia entre los datos obtenidos por el método visual y el Scion Image).

\section{RESULTADOS}

Los valores de la estimación visual y de la estimación por análisis de imá-

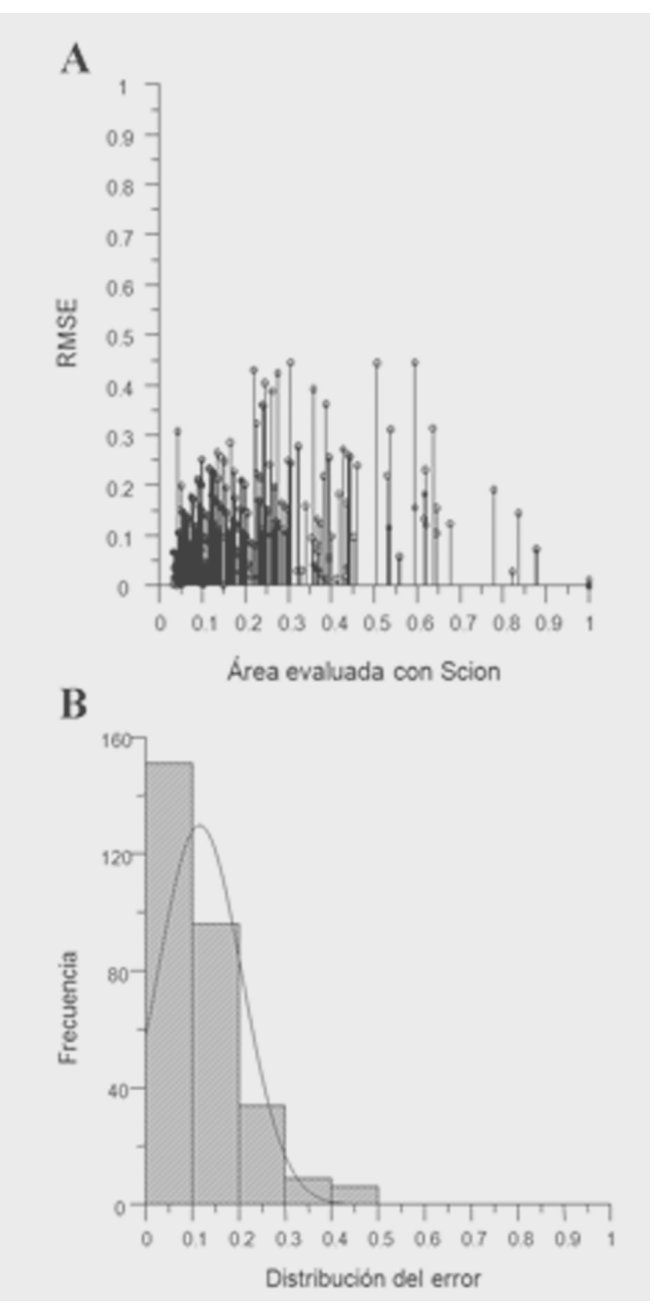

Figura 4.- Análisis de la relación del área evaluada con SCION IMAGE y el RMSE (raíz cuadrada del error medio). (B) Frecuencia de la distribución del error RMSE que cuantifica la diefrencia entre ambos métodos. 
genes digitales muestran una buena correspondencia (Figura 2, $\mathrm{n}=300, \mathrm{r}^{2}=$ $0,79 ; \mathrm{P}<0,001)$. La intercepción y la pendiente fueron significativamente diferentes de 1 y $0(p<0,001)$. La falta de ajuste fue principalmente relacionada al sesgo o a la diferencia media entre lo observado y lo esperado (Usesgo $=$ 0,48 ; Upendiente $=0,13$; Uerror $=0,35$ ).

La distribución del error sugiere que hay una subestimación cuando aplicamos la evaluación visual para determinar el porcentaje de daño (Figura 3). Este error es mayor en los valores intermedios. La desviación estándar de la estimación visual del porcentaje de daño fue de 0,22 y de la evaluación a través del análisis de imágenes digitales fue de 0,19 .

El análisis del error producido al usar el método de la evaluación visual (RMSE) confirmó que la diferencia más grande entre los dos métodos de medida se presentó cuando se evaluaron niveles de daño intermedio, que están entre $0,3-0,7$ (Figura 4A) y no en los casos extremos (0-0,3 ó 0,7-1). Con respecto a la distribución del error (Figura 4B), se observó que el grueso de las diferencias entre los dos métodos fue concentrado al inicio del cuadro de barras $(0-0,2)$. En el 53\% de las muestras evaluadas, se presentó una diferencia en el índice obtenido de 0,1 ; mientras que en el $84 \%$ de las muestras evaluadas existió una diferencia en el índice obtenido de 0,2. Los datos se ajustaron a una curva normal $(\mathrm{n}=300 ;=$ 0,1164; SD = 0,091).

\section{DISCUSIÓN}

Una buena correspondencia de la estimación visual del porcentaje de daño con la evaluación de imágenes digitales ya ha sido reportada en trabajos anteriores en estudios realizados con enfermedades de plantas (6), en la evaluación de daño causado por plagas $(20,21)$ y en la evaluación de lesiones gástricas (22). En cuanto a la distribución del error, éste es mayor en los valores intermedios, lo que se explica a través de la ley de Weber-Fechner, que propone que el ojo humano estima daños altos o bajos con mayor precisión que rangos medios de severidad (23).

En la práctica, la existencia de una distribución normal es de especial importancia. De hecho, se da mayor peso a los resultados correspondientes a estos niveles intermedios, que son difíciles de medir o donde existe mayor probabilidad de cometer errores de apreciación. Esta situación puede ser importante al momento de tomar una decisión para recomendar un control enfocado en mejorar el manejo de la plaga.

Los resultados obtenidos apuntan a que con el procesamiento de imágenes digitales se pueden obtener datos más precisos y se eliminan errores originados por subjetividad. Esto concuerda con resultados obtenidos por otros investigadores $(10,20,24,25)$. 

Marcelo Patiño \& Olivier Dangles

\section{CONCLUSIONES}

El análisis de imágenes digitales se ha utilizado con éxito desde hace algún tiempo atrás en investigaciones relacionadas con agricultura aplicada. Esta es la primera vez que se propone un método cuantitativo, con un procedimiento de evaluación más objetivo y preciso para evaluar niveles de daño provocados por las polillas de la papa $\mathrm{y}$ en particular $T$. solanivora. Se podría también usar en el caso de otros insectos plaga de la papa o de otros tubérculos. Esta metodología deja de lado la percepción humana y da paso al uso de herramientas tecnológicas que están a nuestro alcance y nos permiten tener mayor precisión. La evaluación de los daños mediante el método presentado en este trabajo requiere de un poco más de tiempo y esfuerzo que la evaluación visual pero, en contraparte, sin gastos excesivos (por el uso de acceso libre), se obtienen datos más depurados y confiables. Además, hay que mencionar que el SCION IMAGE tiene otras opciones, como la de poder medir cada una de las partículas dañadas. Esto permitiría cuantificar, por separado, el daño producido por varias plagas en un mismo tubérculo. Esta posibilidad parece muy interesante, en particular en la zona andina, considerando que diferentes especies de polillas de la papa se encuentran en simpatría.

\section{AGRADECIMIENTOS}

Los autores agradecen a la Fundación McKnight por el financiamiento de este estudio a través del proyecto Innovative Approaches for Integrated Pest Managment in the Andes (Grant 09-022). Gracias a Omar Torres por los comentarios y sugerencias que ayudaron a mejorar este manuscrito.

\section{REFERENCIAS \\ BIBLIOGRÁFICAS}

1. BARRAGÁN, A.; ZEDDAM, J. L.; ONORE, G.; CHEVASCO, V.; CHEVASCO, D.; MORTENSEN, E.; PADILLA, J.; ORBE, K. \& LASSO, D. 2005. Problemática de las polillas de la papa en el Ecuador. Memorias del I Encuentro Ecuatoriano de la Papa. Quito, Ecuador, CIP, INIAP-PNRT-Papa, FCAUCE. 8 pp.

2. GALLEGOS, P. \& SUQUILLO, J. 1996. Monitoreo de la polilla de la papa Tecia solanivora (Polvony), en el centro y zonas paperas de frontera de la zona del Carchi. En informe Anual 1996 - 1997. INIAP. Quito, Ecuador.

3. TORRES, F. 1998. Biología y manejo integrado de la polilla centroamericana de la papa Tecia solanivora en Venezuela. Fondo Nacional de Investigaciones Agropecuarias. Fundación para el desarrollo de la Ciencia y Tecnología del estado de Táchira. Serie A-No 14. 57 p. 
4. MENA, A. 2004. Comportamiento y control de polillas de la papa (Tecia solanivora, Symmetrischema tangolias y Phthorimaea operculella) en el Valle de Salcedo, Cotopaxi. Facultad de Agronomía, Universidad Técnica de Cotopaxi, Ecuador. $125 \mathrm{pp}$.

5. GALLEGOS, P. 2003. Problemática de la polilla guatemalteca de la papa Tecia solanivora (Lepidoptera: Gelechiidae) en el Ecuador: antecedentes, desarrollos y perspectivas. En: López, A. (eds.). 2005. III Taller Internacional sobre la polilla guatemalteca de la papa Tecia solanivora. Cartagena de Indias, Colombia. Centro Internacional de la Papa (CIP). 25-36.

6. ARIAS, J.; JARAMILLO, J.; ARÉVALO, E.; ROCHA, N. \& MUÑOZ, L. 1996. Evaluación de la incidencia y severidad del daño de la polilla gigante de la papa Tecia solanivora en el Departamento de Antioquia. Boletín de divulgación. Medellín, Colombia, ICA - CORPOICA. 24 pp.

7. MURCIA, G. \& BARRETO, N. 1997. Evaluación de parámetros para el almacenamiento de semilla de papa (Solanum tuberosum) y mecanismos de control de polilla guatemalteca (Tecia solanivora) durante el mismo. Ministerio de Agricultura y Desarrollo Rural de Colombia, Centro Internacional de Agricultura Tropical y Corporación
Colombiana de Investigación Agropecuaria. $23 \mathrm{pp}$.

8. WIJEKOON, C.P.; GOODWIN, P.H. \& HSIANG, T. 2008. Quantifying fungal infection of plant leaves by digital image analysis using Scion Image software. Journal of Microbiological Methods, 74: 94-101.

9. PITAS, I. 2000. Digital Image Processing Algorithms and Applications. John Wiley \& Sons, Inc.

10. O'NEAL, M.; LANDIS; D. \& ISAACS, R. 2002. An inexpensive, accurate, method for measuring leaf area and defoliation through digital image analysis. Journal of Economic Entomology, 95 (6): 1190-1194.

11. JOHNSON, S. 2001. Indirect impacts on a phytophagous insect of birch: The role of fungal phytopathogens and leaf-mining insects. Tesis de Doctorado submitida a Departamento de Biología de la Universidad de York. 223 pp.

12. AUKER, L. \& OVIATT, C. 2007. Observations on the colonization of the invasive tunicate Didemnum sp. Rhode Island Naturalist, 14 (1): 1 - 4.

13. LEE, D.; O'KEEFE, J.; HOLBROOK, N. \& FIELD, T. 2003. Pigment dynamics and autumn leaf senescence in a New England deciduous forest, Eastern USA. Ecological Research, 18: 677-694.

14. FEILD, T.; LEE, D. \& HOLBROOK, N. 2001. Why leaves turn red in autumn. The role of antho- 

Marcelo Patiño \& Olivier Dangles

cyanins in senescing leaves of Redosier dogwood. Plant Physiology, 127: 566-574.

15. BUSCAGLIA, H. \& VARCO, J. 2002. Early detection of cotton leaf nitrogen status using reflectance. Journal of Plant Nutrition, 25: 2067-2080.

16. BAKR, E. M. 2005. A new software for measuring leaf area, and area damaged by Tetranychus urticae Koch. Journal of Applied Entomology, 129(3): 173-175.

17. PIÑEIRO, G.; PERELMAN, S.; GUERSCHMAN, J. P. \& PARUELO, J. M. 2008. How to evaluate models: Observed vs. predicted or predicted vs. observed? Ecological Modelling, 216: 316-322.

18. PARUELO, J. M., JOBBAGY, E.G., SALA, O.E., LAUENROTH, W.K. \& BURKE, I.C., 1998. Functional and structural convergence of temperate grassland and shrubland ecosystems. Ecological Modelling, 8: 194-206.

19. SMITH, E.P. \& ROSE, K.A., 1995. Model goodness-of-fit analysis using regression and related techniques. Ecological Modelling, 77: 49-64.

20. CHEN, Y. \& WILLIAMS, K. A. 2006. Quantifying Western Flower Thrips (Frankliniella occidentalis Pergande) (Thysanoptera: Thripidae) Damage on Ivy Geranium (Pelargonium peltatum (L.) L'Her ex Ait.) (Geraniaceae Juss.) with
Adobe Photoshop and Scion Image Software. Journal of the Kansas Entomological Society, 79 (1): 83-87.

21. GILBERT, M. \& GRÉGOIRE, J. C. 2003. Visual, semi-quantitative assessments allow accurate estimates of leafminer population densities: an example comparing image processing and visual evaluation of damage by the horse chestnut leafminer Cameraria ohridella (Lep., Gracillaridae). Journal of Applied Entomology, 127: 354 359.

22. KHAN, H. A. 2004. Computerassisted visualization and quantitation of experimental gastric lesions in rats. Journal of Pharmacological and Toxicological Methods, 49: 89-95.

23. HORSFALL, J. G. \& BARRAT, R. W. 1945. An improved grading system for measuring plant diseases. Phytopathology, 35: 655.

24. SU, N. Y. \& MESSENGER, M. T. 2000. Measuring wood consumption by subterranean termites (Isoptera: Rhinotermitidae) with digitized images. Journal of Economic Entomology 93 (2): 412-414.

25. ALCHANATIS，V.; NAVON，A.; GLAZER, I. \& LEVSKI, S. 2000. An image analysis system for measuring insect feeding effects caused by biopesticides. Journal of Agricultural Engineering Research, 77 (3): 289-296. 